Proc. Indian Acad. Sci. (Chem. Sci.), Vol. 96, No. 6, April 1986, pp. 509-521.

(C) Printed in India.

\title{
Electron-electron interactions in polyacetylene $†$
}

\author{
S RAMASESHA \\ Solid State and Structural Chemistry Unit, Indian Institute of Science, Bangalore 560012, India \\ Abstract. Experimental evidence for strong electron-elóctron interactions in polyacetylene is \\ presented. These include (i) observation of a dipole forbidden state below the optical gap, \\ (ii) observation of negative spin densities at sites at which noninteracting models predict zero \\ spin density (iii) vanishing optical gap, in the infinite chain limit, in the closely related \\ symmetrical linear cyanine dyes. To correctly explain these features it is necessary to solve \\ correlated model Hamiltonians. Using diagrammatic valence bond method model exact \\ solutions of correlated models of finite-size systems can be obtained and various physical \\ properties of the low-lying states can be computed. These properties, when extrapolated to the \\ infinite chain limit explain many of the experimental features observed in polyacetylene.
}

Keywords. Electron correlation; valence bond method; polyacetylene; optical gap; spin densities.

\section{Introduction}

Chemists have long been interested in polyenes, both from experimental and theoretical standpoints. For instance, it was known from extrapolations of experimental data on optical gaps of finite polyenes that an infinite polyene should have a finite optical gap of $\sim 2.25 \mathrm{eV}$ (Murrell 1963). There was also much interest concerning the bond alternation in infinite polyenes, since in finite systems, aromatic molecules like benzene had equal $\mathrm{C}-\mathrm{C}$ bond lengths while linear chain molecules like hexatriene had alternating short and long $\mathrm{C}-\mathrm{C}$ bonds. While Coulson (1938) predicted uniform bond lengths for infinite polyenes, Kuhn (1948) predicted dimerization of the carbon chain to account for the finite optical gap. Later calculations by Longuet-Higgins and Salem (1959) showed that the ground state energy per carbon atom varied as $\delta^{2} \ln |\delta|$, for small $\delta$, where $\delta$ is the alternation in the transfer integral. Because of this logarithmic dependence of the ground state energy on the bond alternation, it is to be expected that the ground state is always dimerized, independent of the stiffness of the carbon chain or the strength of electron-lattice coupling constant. This also follows from a more general argument put forth by Peierls (1955) which leads to the conclusion that partially filled one-dimensional bands in the ground state are unstable with respect to a $2 k_{F}$ distortion of the lattice. Polyacetylene, being a half-filled one-dimensional band should undergo a Peierls dimerization in the ground state. Experimentally, x-ray (Fincher et al 1982) and NMR (Yannoni and Clarke 1983) investigations have, in fact, conclusively demonstrated bond alternation of $0.03 \pm 0.01 \AA$ in polyacetylene.

The spurt in theoretical and experimental interest in this area in recent times has been mainly due to the work of Su et al $(1979,1980)$ who showed that bond alternation domain walls first suggested by Pople and Walmsley (1962) can be viewed as solitons and that they constitute elementary excitations of the polyene chain. The soliton states

† Communication No. 349 from the Solid State and Structural Chemistry Unit. 
are located in the middle of the optical gap. Neutral solitons possess spin while charged solitons are nonmagnetic. Electron spin resonance studies on undoped all-trans polyacetylene have indeed shown the presence of paramagnetic defects (Goldberg et al 1979). However, firm evidence for midgap absorption in all-trans polyacetylene is lacking.

All the theories of polyacetylene mentioned above start from the Hückel limit. Electron-lattice interaction leading to alternation in the Hückel transfer or resonance integral is introduced along with a static strain energy contribution. Electron-electron interactions have been considered to be very weak and either neglected or included only in a perturbative scheme (Kivelson and Heim 1982). However, there is considerable experimental evidence in favour of strong electron-electron interactions in polyacetylene. The importance of electron correlations in polyenes has been stressed among others by Cizek et al (1969), Ovchinnikov et al (1972), Honig et al (1975) and Matsen (1978).

It has long been known from extrapolations of experimental data that the optical gap in symmetrical cyanine dyes vanishes in the limit of infinite chain length (Brooker $e t$ al 1945). This is rather puzzling since symmetrical cyanine dyes differ from polyenes only in the end groups and in the limit of infinite chain length this difference should be negligible (Platt 1956). Hence, attributing the optical gap in polyenes entirely to bond alternation is incorrect. The Hückel based models also fail to explain the existence of optically forbidden states below the optical gap in polyenes with four or more double bonds (Hudson et al 1982; Ohmine et al 1978). The observed oscillator strength for the transition across the optical gap is smaller, by at least a factor of two, than that predicted by the Hückel theory (Salem 1966). Recent ESR studies (Thomann et al 1983) have shown the presence of negative spin densities in polyacetylene radicals on sites where the Hückel theory predicts zero spin density. These experimental results clearly bring out the inadequacy of Hückel type models and show the importance of electronelectron interactions in polyacetylene.

Besides explaining the above experimental results, introduction of electron-electron interactions should not result in a uniform ground state as this would contradict the $x$-ray and NMR results on polyacetylene. For this reason, an investigation of the effect of electron-electron interactions on Peierls dimerization also becomes necessary.

Some of the theoretical methods employed for studying correlated models are the Hartree-Fock (HF) and unrestricted Hartree-Fock (UtF) methods, perturbation methods, quantum renormalization group methods, and extrapolations from exact diagonalization of finite systems. The $\mathrm{HF}$ method gives qualitatively the same results as the Hückel model and hence is not suited to explain many of the features discussed above. While the UHF theory is better, it suffers from the drawback that the method does not conserve total spin and consequently predicts spin density wave states even in singlets (Fukutome and Sasai 1982). Perturbation methods work when the correlations are weak and we will demonstrate that the correlations in polyacetylene are strong thereby rendering perturbation method unsuitable in these systems. The quantum renormalization group method (Chui and Bray 1978) is also not accurate since it confines to a rather small part of the Hilbert space spanned by the Hamiltonian. Finite system calculations offer a viable alternative provided reasonably large systems can be solved exactly. Since in the minimum basis Hamiltonian, there are $\mathbf{4}$ possible states for every site, the basis set increases with the system size $N$ as $4^{N}$. To be able to deal with large systems (large $N$ ) the full symmetry of the Hamiltonian should be exploited. The 
correlated model Hamiltonians conserve total spin $\left(S_{\text {tot }}\right)$ besides $S_{\text {tot }}^{Z}$. Therefore, valence bond (VB) functions which are eigen functions of $S_{\text {tot }}$ as well as $S_{\text {tot }}^{z}$ are the natural choice of basis for finite system calculations.

\section{VB method for finite model Hamiltonians}

The Hamiltonian employed for modelling polyacetylene is the Pariser-Parr-Pople (PPP) Hamiltonian given by

$$
\begin{aligned}
& H=H_{t}+H_{\text {corr }} . \\
& H_{t}=\sum_{p, \sigma} t_{p}\left(a_{p, \sigma}^{*} a_{p+1, \sigma}+a_{p+1, \sigma}^{*} a_{p, \sigma}\right), \\
& H_{\text {corr }}=U \sum_{p} \hat{n}_{p}\left(\hat{n}_{p}-1\right) / 2+\sum_{p>p^{\prime}} V_{p p^{\prime}}\left(\hat{n}_{p}-1\right)\left(\hat{n}_{p}-1\right),
\end{aligned}
$$

where $a_{p, \sigma}^{*}\left(a_{p, \sigma}\right)$ creates (annihilates) an electron with spin $\sigma$ in the orbital at site $p$. The transfer integral $t_{p}$ is estimated to be $2.4 \pm 0.17 \mathrm{eV}$ for double and single bonds for polyacetylene. $U$ is the on-site correlation energy which from gas phase ionization and electron affinity data for carbon is $11.26 \mathrm{eV}$. Since polyacetylene chains are semiconducting, the screening of correlations is not very effective and long-range interactions will also have to be included besides the on-site interactions $U$. The second term in $H_{\text {corr }}$ corresponds to such interactions and is given by the Ohno formula (Ohno 1964)

$$
V_{p p^{\prime}}=U\left(1+0.6117 r_{p p^{\prime}}^{2}\right)^{-1 / 2} \text {, }
$$

which interpolates between $U$ for $p=p^{\prime}$ and $\pm\left(e^{2} / r_{p p^{\prime}}\right)$ for $\left|p-p^{\prime}\right| \rightarrow \infty$. The Hamiltonian with only on-site interactions is also useful in these studies, since for uniform $t_{p}$, the model has been exactly solved for the infinite chain (Lieb and Wu 1968) and provides a stringent test of extrapolations. Since we are interested in comparing results between correlated and uncorrelated models for the same lattice stiffness and electron-lattice interactions, we have not considered the elastic term.

A normalized singlet va function can be generated by operating on the vacuum state with $a_{p \alpha}^{*} a_{p \beta}^{*}$ for a doubly occupied site $p$ and by $\left(a_{p \alpha}^{*} a_{\phi \beta}^{*}-a_{p \beta}^{*} a_{a \alpha}^{*}\right) / \sqrt{ } 2$ for singlet pairing of electrons on sites $p$ and $q$. A given $\mathrm{VB}$ function can be conveniently represented as a diagram with dots ( $)$ at empty sites, crosses $(x)$ at doubly oocupied sites and lines between pairs of sites at which electrons are singlet paired (figure 1). These diagrams in turn can be coded in the binary system by associating two bits per site (Ramasesha and Soos 1984a). The states of the bits are ' 00 ' for any empty site, ' 10 ' for a site at which a line begins, '01' for a site at which a line ends and ' 11 ' for a site that is doubly occupied. The Rumer-Pauling rule states that linear independence and completeness can be achieved by retaining all diagrams without intersecting lines, on arranging the $N$ sites at the vertices of a polygon. This rule makes the binary representation of a vB diagram unique.

All the vB diagrams with a given total spin $S$, a given number of sites $N$ and a given number of electrons $N_{e}$ on the $N$ sites can now be generated in an increasing sequence of integers that represent them, by simply examining whether the bit pattern of an 

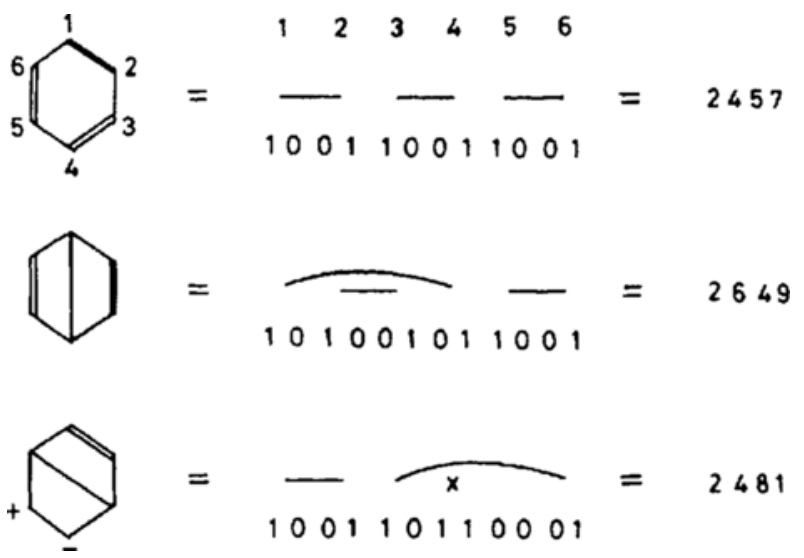

Figure 1. Typical singlet VB diagrams for six electrons on six sites and their bit representation.

integer in question corresponds to a VB diagram of the given characteristics. The total number $P_{s}(2 n, N)$ with spin $S, N_{e}=2 n \leqslant N$ is given by

$$
P_{s}(2 n, N)=\frac{2 S+1}{N+1} \frac{[(N+1) !]^{2}}{(n+S+1) !(n-S) !} \times \frac{1}{(N-n-S) !(N+S+1-n) !}
$$

$P_{s}(2 n, N)$ is much smaller (usually by a factor of $\sim 3-4$ ) than the complete basis formed by Slater determinants. The size of the basis can further be reduced by taking into account electron-hole and spatial symmetries that may exist in the Hamiltonian.

The yв basis is diagonal in the correlation part of the Hamiltonian (1) but is offdiagonal in the transfer part. The exact matrix representing the model Hamiltonian can be obtained by operating on the vB functions by the Hamiltonian (Mazumdar and Soos 1979). The matrix elements are defined by

$$
H|k\rangle=\sum_{j} h_{k j}|j\rangle,
$$

where $|i\rangle$ represents a vB function. Determining the column index in $h_{k j}$ is made efficient since the vв diagrams are arranged as increasing integers that allow binary searching. The Hamiltonian matrix $h$ is non-symmetric, because the vB basis is nonorthogonal. As with any configuration interaction calculations, the matrix $h$ is sparse. We are interested only in a few low-lying states, so that taking advantage of the sparseness, it is possible to deal with upto $\sim 60000 \times 60000$ matrices on a supermini computer like vAX 11/780. The low-lying eigen values and corresponding eigen vectors are obtained by coordinate relaxation and deflation where necessary (Ramasesha and Soos 1984a).

Calculation of matrix elements between correlated states first requires that the correlated state be normalized, i.e., we need to evaluate

$$
R=\sum_{k, k^{\prime}} C_{k^{\prime} n} C_{k n} S_{k, k^{\prime}}
$$

The overlap matrix $S_{k, k^{\prime}}$ is of the same order as the basis set. Bruteforce evaluation of 
$S_{k^{\prime} k}$ is very time consuming. On the other hand, using charge orthogonality of vB functions, the basis can be rearranged such that $S_{k^{\prime} k}$ is block diagonal with blocks of the same size being identical (figure 2) (Ramasesha and Soos 1984b). We are now left with the simpler task of evaluating the overlap matrix elements within a block and that too only once for a block of a given size. The overlap matrix elements are calculated using Pauling's island counting scheme (Pauling 1933). For instance, for a 10 electrons on 10 sites problem, the singlet basis has 19404 diagrams. The overlap matrix of this basis when written in the block-diagonal form has one $42 \times 42$ block and several $14 \times 14,5 \times 5,2 \times 2$ and $1 \times 1$ blocks. The overlap matrix is calculated only once for the $14 \times 14,5 \times 5$ and $2 \times 2$ blocks even though they appear several times.

Matrix element calculation for operators diagonal in the $\mathrm{VB}$ representation is straightforward. An example of such an operator is the dipole moment operator. The transition dipole between eigen states $\left|\psi_{n}\right\rangle$ and $\left|\psi_{m}\right\rangle$ of the Hamiltonian (1) is given by

$$
\mathbf{M}_{n m}=\frac{1}{R}\left\langle\psi_{n}|\mu| \psi_{m}\right\rangle=\frac{1}{R} \sum_{k k^{\prime}} C_{k^{\prime} m} C_{k n} \mu_{k} S_{k^{\prime} k}
$$

The matrix elements of operators which are not diagonal in the VB representation but conserve total spin can also be calculated easily. However, in such calculations the resultant state obtained by the effect of such operators on the eigen state of the Hamiltonian requires reordering into the block-diagonal sequence. An example of such an operator is the operator for obtaining bond orders,

$$
b_{p}=\sum_{\sigma}\left(a_{p+1, \sigma}^{*}, a_{p, \sigma}+a_{p, \sigma}^{*} a_{p+1, \sigma}\right) .
$$

Matrix elements of operators that do not conserve total spin and also do not transfer electrons between sites such as the local spin density operator $s_{p}^{z}$, the spin-spin

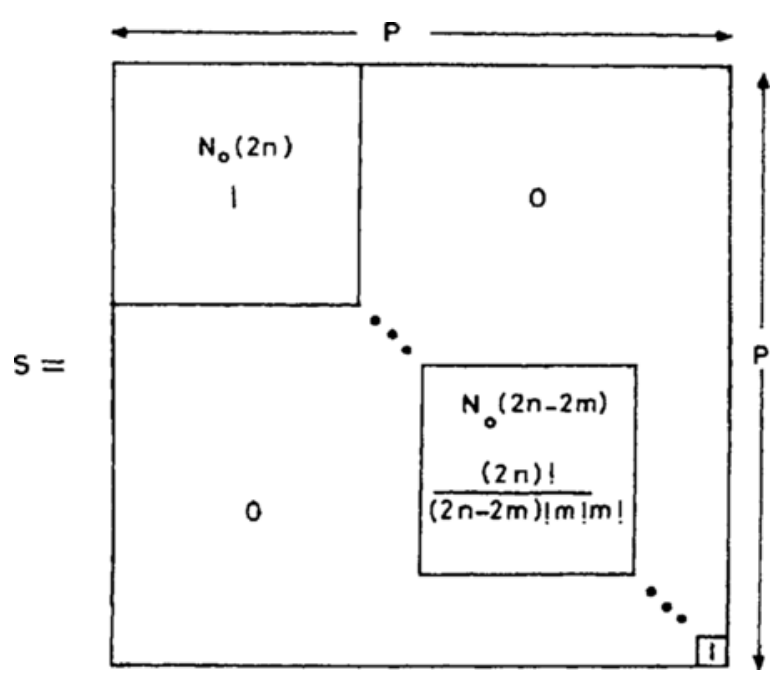

Figure 2. Overlap matrix $S$ in the block-diagonal representation. $N_{0}(2 r)$ are the covalent singlets for $2 r$ electrons on $2 r$ sites. The expression below $N_{0}(2 r)$ gives the number of times a block of $N_{0}(2 r)$ repeats. $P$ is the size of the total basis. 
correlation operator $s_{p}^{z} s_{q}^{z}$ can also be handled in this scheme by more general techniques (Ramasesha and Soos 1985).

Evaluation of such a variety of matrix elements between model exact eigen states of Hamiltonians helps in a better comparison of the model with a wide range of experimental results that exist for polyacetylene.

\section{Results and discussion}

Our method of obtaining results for the infinite chain is based on extrapolation of the model exact finite system results to the infinite limit. Although it is known that complete configuration interaction calculation is size consistent, there is no a priori theoretical basis for extrapolations. Therefore, to have confidence in the extrapolation procedures, it is necessary that we compare the extrapolated results with exact infinite chain results, wherever they exist. Fortunately, in the present case, exact ground state energy per site as well as the optical gap of infinite uniform Hubbard chains $\left(V_{p p^{\prime}}=0\right.$ for $\left.p \neq p^{\prime}\right)$ is known (Lieb and Wu 1968). Since $U=4 t$ in the Hubbard model is the most difficult regime in the parameter space for approximate methods, we compare our extrapolated results for this case with the exact results for the ground state energy per site (figure 3 ) as well as the optical gap (figure 4). The extrapolated value for the ground state energy per site is $-0.573 \pm 0.005$ while the exact result is -0.57373 , in units of $t$. The optical gap from extrapolations is $1 \cdot 20 \pm 0-1$ while the exact gap is $1 \cdot 2867$, again in units of $t$. These

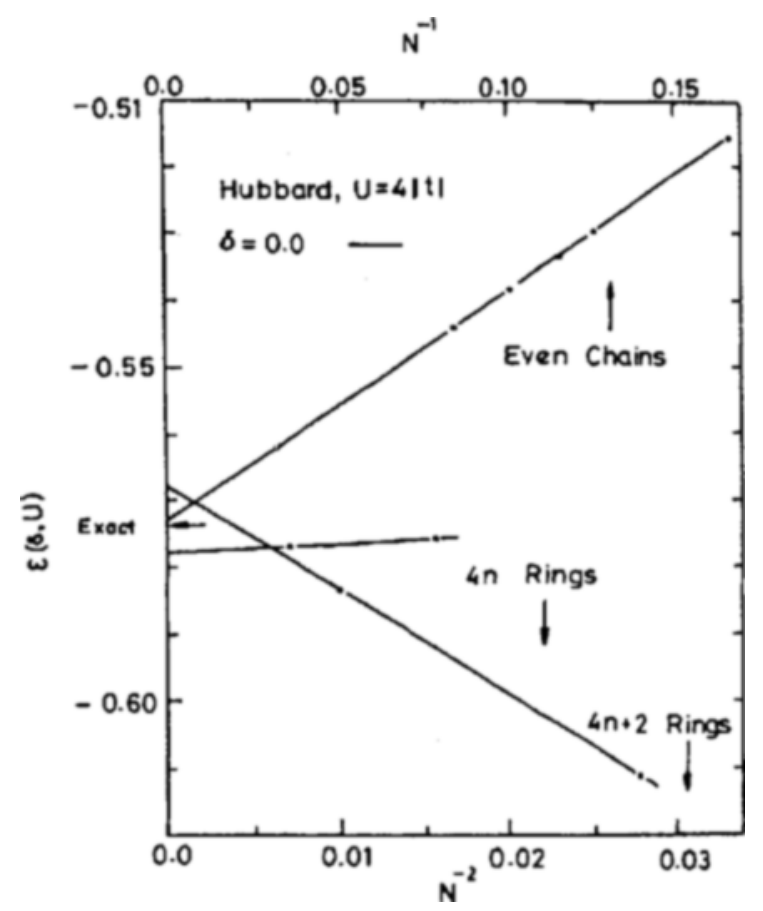

Figure 3. Ground state energy per site $\varepsilon(0, U)$ in units of $t$ for the uniform Hubbard model, vs $N^{-1}$ for chains and $N^{-2}$ for rings. 
results, besides giving confidence in the extrapolation methods also show that in such schemes, ground state energy per site has smaller error than the optical gap. This is important since in studying the effect of correlations on dimerization of the half-filled one-dimensional band, we would be comparing the difference in ground state energies per site between $\delta=0$ and $\delta \neq 0$ cases, which are rather small for small $\delta$.

Although exact results do not exist for $\delta \neq 0$ case of the Hubbard model, our results show that the optical gap widens by $\sim 0.20$ (in units of $t$ ), in clear disagreement with the meanfield result (Bychkov et al 1966) which predicts the gap to widen by only 0.03 (in units of $t$ ) on introducing this dimerization. This once again illustrates that the predictions of mean field theory should be viewed with caution.

In the case of the full Hamiltonian of (1), there are no known exact results for the infinite system. However, we can compare exact results for finite chains with experimental quantities since finite chains correspond to small polyenes. Using the same values of $U, t$ and $\delta$, we find our results for all-trans $N=8$ and $N=10$ chains to be in very good agreement with the spectroscopic data for octatetraene and decapentaene (table 1). The optical gaps in pentadienyl and heptatrienyl ions from the theory are $3.456 \mathrm{eV}$ and $2.79 \mathrm{eV}$ while experiments yield a gap of $3.42 \pm 0.12 \mathrm{eV}$ and $2.88 \pm 0.04 \mathrm{eV}$ respectively. The correlation diagram in figure 5 shows that the correlations split the degenerate excited states in the Hückel model. States with electron-hole symmetry in which covalent vB diagrams appear with nonzero coefficients are lowered relative to the states with opposite electron-hole symmetry in which the covalent diagrams have strictly zero weight. In the limit of very large correlations, all the

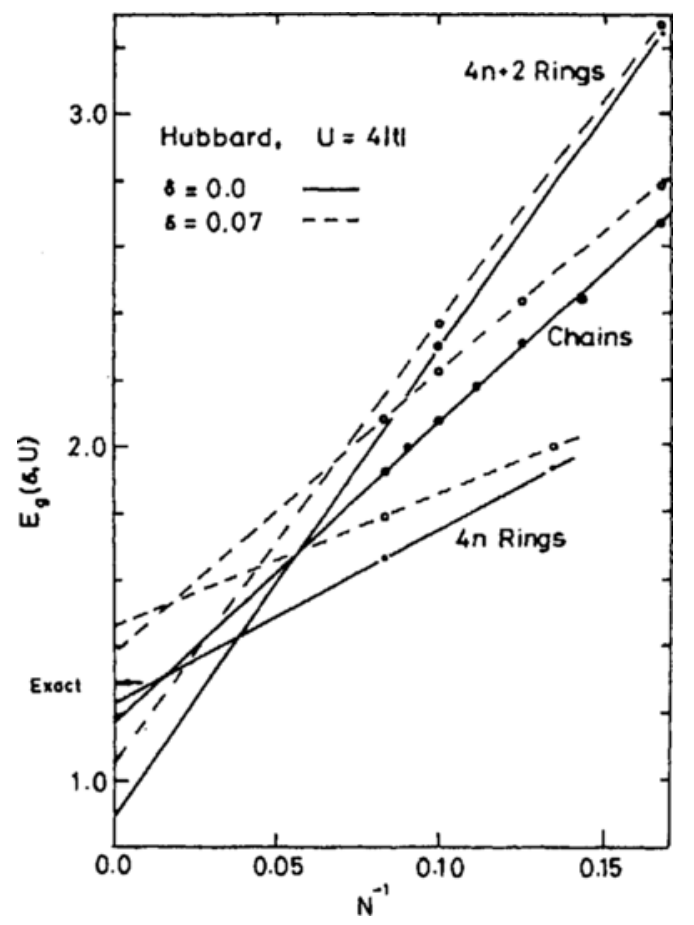

Figure 4. Optical gap in units of $t$ vs $N^{-1}$ for uniform and dimerized Hubbard chains. The exact result is for the uniform Hubbard model. 
Table 1*. Exact PPP and experimental excitation energies of all-trans octatetra $\left(N=N_{\varepsilon}=8\right)$ and decapentaene $\left(N=N_{e}=10\right)$. Covalent and ionic labels designate electron-hole symmetry. The lowest dipole-allowed excitation is underlined.

\begin{tabular}{|c|c|c|c|c|c|}
\hline \multicolumn{2}{|c|}{ State } & \multicolumn{2}{|c|}{ Decapentaene } & \multicolumn{2}{|c|}{ Octatetraene } \\
\hline$S=0$ & $S=1$ & PPP (eV) & Experimental (eV) & PPP $(\mathrm{eV})$ & Experimental (e \\
\hline \multirow[t]{4}{*}{$1^{1} A_{p}$ (cov) } & & - & - & 一 & - \\
\hline & $1{ }^{3} B_{\mathrm{u}}$ (cov) & 1.756 & - & 1.920 & $2 \cdot 1$ \\
\hline & $1^{3} A_{g}$ (cov) & $2 \cdot 566$ & - & 2.929 & - \\
\hline & $2{ }^{3} B_{4}$ (cov) & 3.388 & - & 3.844 & - \\
\hline $2^{1} A_{1}(\operatorname{cov})$ & & $3 \cdot 404$ & $3 \cdot 10$ & 3.775 & 3.59 \\
\hline $1^{\prime} B_{\mathrm{x}}$ (cov) & & $4 \cdot 210$ & - & $4 \cdot 718$ & - \\
\hline $1^{1} B_{\mathrm{w}}$ (ion) & & $\underline{4.234}$ & $\underline{4 \cdot 02}$ & $\underline{4.561}$ & $\underline{4 \cdot 40}$ \\
\hline \multirow[t]{2}{*}{$2^{2} B_{4}$ (cov) } & & $\overline{5 \cdot 323}$ & - & $\overline{6.644}$ & $=$ \\
\hline & $1{ }^{3} A_{p}$ (ion) & 5.494 & - & 5.977 & - \\
\hline \multirow{2}{*}{$1^{2} A_{q}($ ion $)$} & & 5.589 & 一 & 6.086 & 一 \\
\hline & $1^{3} B_{x}$ (ion) & 6.458 & - & 7.072 & - \\
\hline
\end{tabular}

* From Ramasesha and Soos (1984b); cov = covalent; ion = ionic.

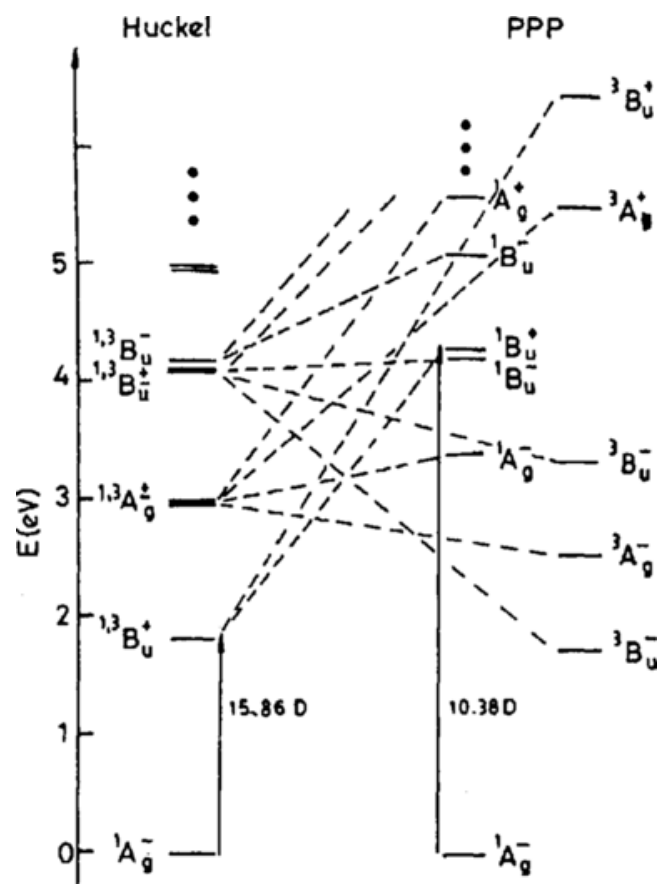

Figure 5. Correlation of Hückel and PPP excitation energies for all-trans decapentaene alternation $\delta=0.07$, also shown is the theoretical transition moment for the lowest d. transition. 
covalent states descend below the optical gap and form spin-wave excitations of the chain. Figure 5 shows that for decapentaene, with our parameters, there exist two optically forbidden states below the optical gap. Existence of the $2 A_{0}^{1}$ state in decapentaene is experimentally confirmed from gas phase optical studies and is located $3.10 \mathrm{eV}$ above the ground state, to be compared with the model exact value of $3.404 \mathrm{eV}$. Figure 5 also indicates that the transition moment for the lowest optical transition is lowered substantially from the Hückel value and is in much better agreement with experiments (Soos and Ramasesha 1983a). It appears that our parameters slightly overestimate the correlations. Nonetheless, our calculations convincingly prove that correlations are very strong in polyacetylene and cannot be neglected or even included reliably within a perturbative scheme.

The extrapolations from finite-size system to the infinite system has been done for both rings and chains (Soos and Ramasesha 1984). Ideally, the ring and chain extrapolations for $N \rightarrow \infty$ should give the same values. However, because of small system sizes the two extrapolations do not converge to the same value. The mismatch in these extrapolations gives an estimate of the error involved in these procedures.

Figure 6 shows the extrapolated optical gap for a single strand of polyacetylene. The $\delta=0$ extrapolation is also indicated on the figure to show that most of the optical gap arises from electron correlations rather than from dimerization. The extrapolated gap of $2.8 \pm 0.2 \mathrm{eV}$ should be compared with the observed solid state gap of $1.9 \mathrm{eV}$. Considering that the gas phase optical gap will be red shifted by $\sim 0.5 \mathrm{eV}$ in the solid state, we find that the correlations we have assumed slightly overestimate the correlations in polyacetylene.

It is well known that correlations introduce negative spin densities on sites where the $\mathrm{HF}$ theory predicts zero spin density (McConnel and Chesnut 1957). Indeed, as already mentioned, negative spin densities have been observed in radicals of all-trans

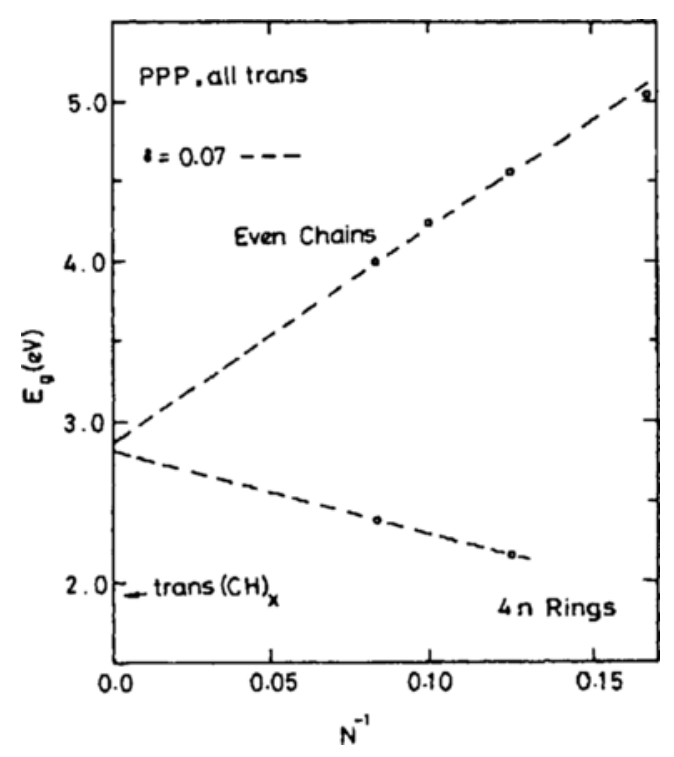

Figure 6. Optical gap $E$, vs $N^{-1}$ for chains and rings in the correlated model. 
polyacetylene (Thomann et al 1983). In figure 7, we have plotted the ratio of total negative to positive spin density, calculated from the correlated ground state wave function, versus inverse system size (Soos and Ramasesha 1983b). The same ratio calculated for uniform Heisenberg chains with odd numbers of spins is shown to delineate the large $U$ limit. Experimentally observed ratios for polyacetylene radicals with $N \sim 50$ is also shown. The comparison between the theoretical and experimental results once again confirms our view that we have only slightly overestimated the correlations in polyene chains.

Figure 8 shows the dependence on system size of optical gaps of uniform chains, ions with odd numbers of carbon atoms and cyanine dyes, as well as of covalent gaps of radicals. The optical gaps of uniform chains fall on the same line for both even and odd chains. This shows that the mid-gap state does not exist in the correlated models. This is to be expected since the optical gap is primarily due to correlations and only weakly dependent on dimerization. Cyanine dyes are similar to ions of the carbon chain with odd number of carbon atoms and show vanishing optical gap in the finite chain limit. In both the systems, in the infinite chain limit the ground and optically excited states can be described as incommensurate charge density waves shifted in phase and therefore they have the same energy. The covalent gap also vanishes for uniform chains, consistent with the result for uniform Heisenberg chains where the ground state singlet and lowest excited singlet are degenerate to $O(1 / N)$ (Ramasesha and Soos 1983).

Effect of correlations on the dimerization of one-dimensional half-filled bands has been of considerable interest. Qualitative arguments based on the concept of resonance put forth by Mazumdar and Dixit $(1983,1984)$ leads to the conclusion that on-site correlations tend to stabilize the dimerized state. This is borne out by their calculations on rings of six and ten carbon atoms. Mazumdar and Campbell (1985) have extended this argument to long-range coulomb interactions. Soos and Ramasesha (1984) have compared the stabilization of the infinite dimerized chain in the presence of both onsite and extended range interactions, obtained from extrapolations of exact results for

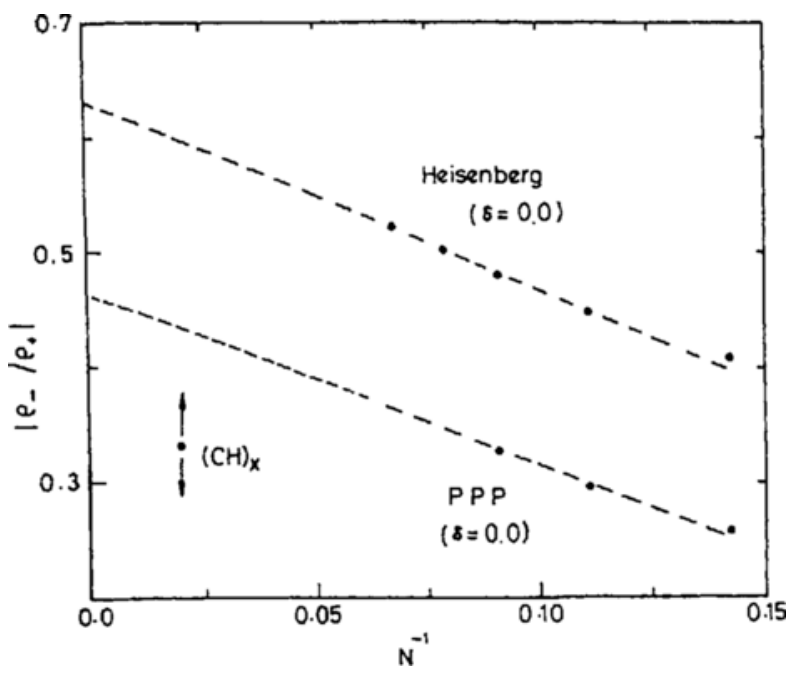

Figure 7. Absolute ratio of total negative to positive spin density vs $N^{-1}$ for Heisenberg spin chains and the PPP Hamiltonian. $(\mathrm{CH})_{x}$ value is from Thomann et al (1983). 


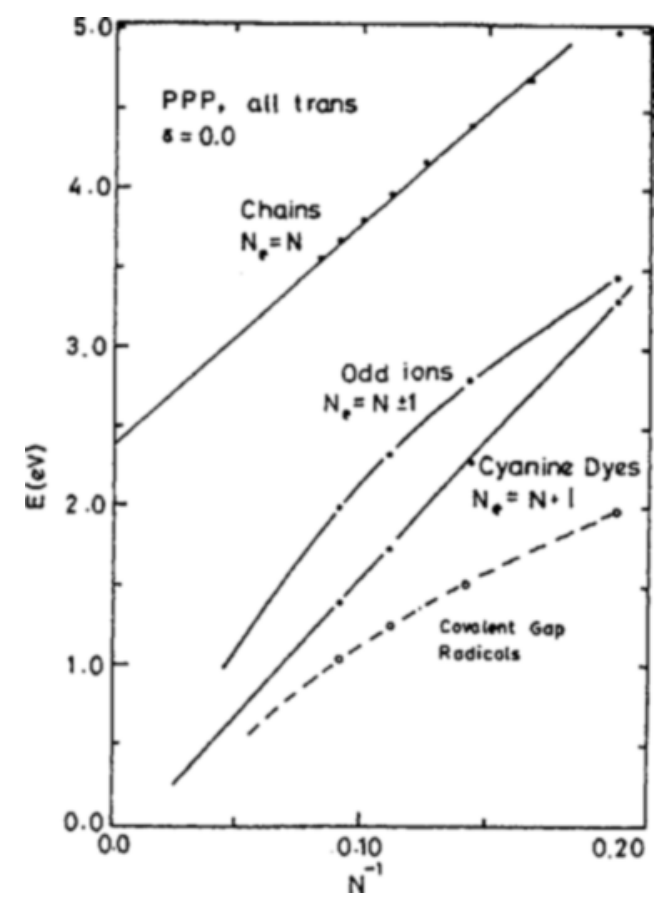

Figure 8. Optical gaps vs $N^{-1}$ of uniform PPP chains, ions of odd segments and linear cynanine dyes. The covalent gap of radicals is dipole forbidden.

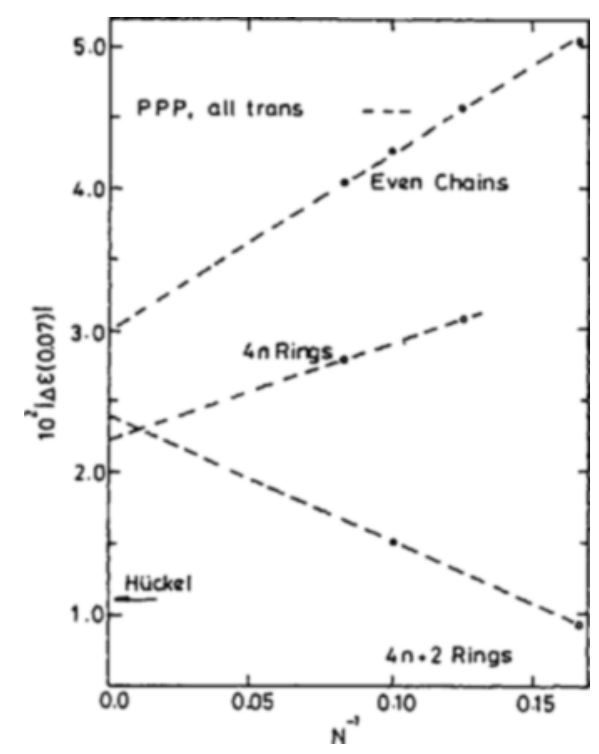

Figure 9. $\Delta \varepsilon(\delta)=\varepsilon(\delta)-\varepsilon(0)$ where $\varepsilon(\delta)$ is the ground state energy per site for alternation $\delta$ in the PPP model, vs $N^{-1}$. Hückel value for the infinite chain is indicated by the arrow. 
both rings and chains of upto twelve carbon atoms, with the stabilization in the Hückel model (figure 9). It is seen that correlations indeed stabilize dimerization. Therefore existence of correlations is consistent with both the observed dimerization and the other experimental results already discussed. However, what remains to be included in a more complete theory of polyacetylene is the interchain interactions and effects of dopants. Further work in this direction is currently in progress.

The diagrammatic vB method together with bit representation provides a powerful technique for exact diagonalization of diverse types of finite model Hamiltonians. It can also be used together with different types of approximation schemes such as Lanczo's scheme (Roomany et al 1980) or the quantum Monte Carlo method (Hirsch et al 1982). Work in these directions is in progress. However, the success of finite system calculations in predicting the properties of infinite systems may not be as dramatic as in the simple case of polyacetylene unless reliable scaling methods are developed for quanturn systems.

\section{References}

Brooker L G S, Sklar A L, Cressman H W J, Keyes G H, Smith L A, Sprague R H, Van Lare E, Van Zandt G, White F L and Williams W W 1945 J. Am. Chem. Soc. 671875

Bychkov L A, Gorkov L P and Dzyaloshinskii IE 1966 Zh. Eksp. Teor. Fiz. 50738 [1966 Soc. Phys. -JETP 23 489]

Chui S T and Bray J W 1978 Phys. Rev. B18 2426

Cizek J, Paldus J and Srobkova L 1969 Int. J. Quant. Chem. 3149

Coulson C A 1938 Proc. R. Soc. London A164 383

Fincher Jr. C R, Chen C E, Heeger A J and MacDiarmid A G 1982 Phys. Rev. Lett. 48100

Fukutome H and Sasai M 1982 Prog. Theor. Phys, 6741

Goldberg I B, Crowe H R, Newman P R, Heeger A J and MacDiarmid A G 1979 J. Chem. Phys. 701132

Hirsch J E, Sugar R L, Scalapino D J and Blankenbecler R 1982 Phys. Rev. B26 5033

Honig B, Warshel A and Karplus M 1975 Acc. Chem. Res. 892

Hudson B S, Kohler B E and Schulten K 1982 in Excited states (ed.) E C Lim (New York: Academic Press) vol. 6

Kivelson S and Heim D 1982 Phys, Rev, B26 4278

Kuhn H 1948 Helv. Chim. Acta 311441

Leib E H and Wu F Y 1968 Phys. Rev. Lett. 201445

Longuet-Higgins H C and Salem L 1959 Proc. R. Soc. London A25 172

Matsen F A 1978 Acc. Chem. Res. 11387

Mazumdar S and Campbell D K 1985 Phys. Rev. Lett. 552067

Mazumdar S and Dixit S N 1983 Phys. Rev. Lett. 51292

Mazumdar S and Dixit S N 1984 Phys. Rev. B29 1824

Mazumdar S and Soos Z G 1979 Synth. Metals 177

McConnel H M and Chesnut D B 1957 J. Chem. Phys. 27984

Murrel J N 1963 The theory of electronic spectra of organic molecules (London: Wiley)

Ohmine I, Karplus M and Schulten K 1978 J. Chem. Phys. 682298

Ohno K 1964 Theor. Chim. Acta 2219

Ovchinnikov A A, Ukrainski I I and Kventsel G V 1972 Usp. Fiz. Nauk. 10851 [1973 Sov. Phys. Usp. 15 575]

Pauling L 1933 J. Chem. Phys. 1280

Peierls R E 1955 Quantum theory of solids (Oxford: Clarendon) p. 109

Platt J R 1956 J. Chem. Phys. 2580

Pople J A and Walmsley S H $1962 \mathrm{Mol}$. Phys. 515

Ramasesha S and Soos Z G 1983 Solid State Commun. 46409

Ramasesha S and Soos Z G 1984a Int. J. Quant. Chem. 251003

Ramasesha S and Soos Z G 1984b J. Chem. Phys. 803278

Ramasesha S and Soos Z G 1985 Phys. Rev. B32 5368 
Roomany H H, Wyld H W and Holloway L E 1980 Phys. Rev. D21 1557

Salem L 1966 The molecular orbital theory of conjugated systems (New York: Benjamin)

Soos Z G and Ramasesha S 1983a Chem. Phys. Lett. 10134

Soos Z G and Ramasesha S 1983b Phys. Rev. Lett. 512374

Soos Z G and Ramasesha S 1984 Phys. Rev. B29 5410

Su W P, Schrieffer J R and Heeger A J 1979 Phys. Rev. Lett. 421698

Su W P, Schrieffer J R and Heeger A J 1980 Phys. Rev. B22 2099

Thomann H, Dalton L R, Tomkiewicz Y, Shiren N S and Clarke T C 1983 Phys. Rev. Lett. 50553

Yannoni C S and Clarke T C 1983 Phys. Rev. Lett. 511191 